\title{
AVALIAÇÃO DO DESEMPENHO LOGÍSTICO DO TRANSPORTE RODOVIÁRIO DE MADEIRA DE ÁREAS DE FOMENTO FLORESTAL COM O USO DE REDE DE PETRI ${ }^{1}$
}

\author{
Carlos Cardoso Machado², Raiane Ribeiro Machado ${ }^{3}$, Edson Arlindo Silva ${ }^{3}$ e Marcelo Freitas \\ Gomes de Souza ${ }^{4}$
}

\begin{abstract}
RESUMO - Neste estudo, buscou-se aplicar o modelo de avaliação do desempenho logístico de cadeias produtivas (ADELCAP), baseado nos tempos dos elementos do ciclo operacional do transporte de madeira de áreas de fomento de uma empresa produtora de celulose do Estado do Espírito Santo, objetivando apontar meios de otimizar os recursos físicos para garantir um fluxo de abastecimento ininterrupto e apropriado volume de entrada de madeira nos depósitos regionais. O ciclo operacional de transporte de madeira é ordenado pelas atividades de carregamento de madeira no campo, viagem carregada, descarregamento no depósito e viagem vazia. Utilizou-se a Rede de Petri Temporal, por trabalhar com dois tempos - duração de sensibilização. Realizaramse simulações com os softwares JARP e ARP, a fim de simular, analisar e avaliar o desempenho operacional. As variações empregadas em cada cenário se devem à qualidade da estrada, sob as condições atuais quanto à geometria vertical e horizontal e ao quociente de irregularidade, em cada depósito para os diferentes tipos de veículo e forma de carregamento da madeira adotado. Nas regiões de topografia acidentada, a melhoria das estradas alterando seu nível de qualidade tem maior influência para o aumento do desempenho dos veículos, e os veículos 4×2 e 6×2 apresentaram os melhores resultados. Nas regiões de planícies, a escolha do veículo tem maior influência no aumento do desempenho do transporte, já que a topografia e qualidade das estradas atuais já contribuem para isso. Nesses casos, a relação tonelada transportada por distância e tempo será maior para os veículos com capacidade de carga líquida maior.
\end{abstract}

Palavras-chave: Desempenho logístico, cadeia produtiva de madeira e simulação.

\section{EVALUATION OF THE WOOD HAULING LOGISTIC PERFORMANCE IN FARM FOREST AREAS USING PETRI NET}

\begin{abstract}
This study aimed at applying the model of logistic performance evaluation of productive chains (ADELCAP), based on the times of the elements of the operational cycle of wood transport from areas of fomentation of a cellulose producing company in the State of Espírito Santo, to indicate means of optimizing physical resources, guaranteeing a flow of uninterrupted provisioning and appropriate volume of wood entrance in the regional deposits. The operational cycle of wood transport is organized by the activities of wood loading in the field, wood hauling, wood unloading in the deposit and unhauling. The Temporal Petri Net was used, because it works with two times - sensitization duration. Simulations were carried out with the ARP and ARP software systems, in order to simulate, analyze and evaluate the operational performance. The variations employed in each scenery are due to the quality of the highway, under the current conditions, as for the vertical and horizontal geometry and the irregularity quotient, in each deposit, for the different types of vehicle and form of the adopted wood loading. In the areas of uneven topography, the improvement of the highways altering their quality level has a higher influence for the increase in the performance of the vehicles, and Toco and Truck presented better results. In the areas of plains, the choice of the vehicle has a higher influence
\end{abstract}

\footnotetext{
${ }^{1}$ Recebido em 03.02.2009 e aceito para publicação em 23.06.2009.

${ }^{2}$ Departamento de Engenharia Florestal da Universidade Federal de Viçosa (UFV). E-mail: <machado@ufv.br>.

${ }_{3}^{3}$ Departamento de Administração da UFV - Campos Rio Paranaiba. E-mail: <raianemachado@ tufv.br>e <edsonsilva@ufv.br>.

${ }^{4}$ Graduação em andamento em Agronomia. UFV Rio paranaiba, Brasil. E-mail:<mgomez@terra.com.br>.
} 
in the increase of the performance of the transport, since the topography and the quality of the current highways already contribute to the performance. In these cases, the relation between transported ton and distance and time will be higher for the vehicles with higher capacity of liquid load.

Keywords: Logistic performance, wood chain supply and simulation.

\section{INTRODUÇÃO}

O transporte mobiliza o produto entre diferentes estágios da cadeia de suprimento. Assim, como outros fatores-chave da cadeia de suprimento, ele exerce grande influência tanto na responsividade quanto na eficiência (CHOPRA e MEINDL, 2004). A operação de transporte florestal consiste na movimentação de madeira dos pátios ou das margens das estradas florestais até o local de consumo (MACHADO et al., 2000), além do transporte da madeira advinda de áreas de fomento até depósitos de entrega das fábricas. O transporte rodoviário é o mais utilizado no Brasil para movimentação de cargas, contribuindo, de maneira significativa, na composição dos custos de diversos segmentos da economia (VELLOSO et al., 1997). A importância do caminhão como meio de transporte deve-se não só ao volume de carga a ser transportado, mas a versatilidade ou facilidade de deslocamento e interligação entre pontos de origens e destinos, situados em quase toda a superfície terrestre.

A crescente necessidade de matéria-prima florestal, aliada à dificuldade de aquisição de novas áreas próximas às unidades industriais, tendo em vista a valorização da terra, tem levado as empresas do setor florestal a buscar novas alternativas para o reflorestamento, já que a sustentabilidade e competitividade da indústria florestal brasileira dependem da expansão da base florestal. Além disso, é evidente a expectativa de aumento da pressão da sociedade sobre as empresas detentoras de grandes maciços florestais homogêneos (CANTO, 2006).

Em Machado et al. (2006) foi constatada a eficiência do uso de Rede de Petri Temporal como ferramenta de modelagem, simulação, análise e avaliação de desempenho da cadeia logística de suprimento rodoviário de madeira própria, em que foi possível diagnosticar o atual nível de desenvolvimento logístico do transporte em questão, através de um modelo desenvolvido utilizando-se a Rede de Petri Temporal. A Rede de Petri é uma ferramenta de modelagem matemática e gráfica com atividades concorrentes e assíncronas que permitem a análise de sistemas complexos de produção, os quais são difíceis de serem modelados por técnicas tradicionais como Filas de Espera, Cadeias de Markov e Redes Perts. A partir deste estudo, percebeu-se a necessidade de realização da mesma análise para o caso de madeira oriunda de fomento florestal.

Dessa forma, o objetivo deste trabalho foi avaliar, através de simulações com Rede de Petri, o impacto da melhoria nas estradas no transporte de madeira de áreas de fomento, levando-se em consideração o tipo de veículo adotado, a forma de carregamento da madeira no campo e a qualidade das estradas, através dos índices de geometrias vertical e horizontal e de quociente de irregularidade.

\section{MATERIAL E MÉTODOS}

\section{1. Áreas de Estudo}

O estudo foi desenvolvido com os grupos dos produtores rurais que possuíum contratos de fomento florestal com a Aracruz Celulose, no Estado do Espírito Santo. Os fomentados foram divididos em grupos, de acordo com o depósito de entrega da madeira ao qual estava vinculado seu contrato. Sendo eles denominados Acampamento (AC), Araguaia (AR), Conceição da Barra (CB), Fábrica (FB) e Santa Teresa (ST), localizados nos respectivos Municípios de Colatina, Marechal Floriano, Conceição da Barra, Aracruz e Santa Teresa.

\subsection{Descrição da cadeia logística de transporte de madeira de áreas fomentadas}

O suprimento logístico da fábrica de celulose se dá pela madeira advinda de reflorestamento próprio da empresa ou de fomento dos produtores rurais regionais. Assim, após a colheita a madeira segue seu ciclo operacional logístico de transporte através das atividades de carregamento manual ou mecanizado da madeira; descolamento do veículo carregado até o respectivo depósito; descarregamento da madeira no pátio do depósito, sempre mecanizado; e deslocamento do veículo vazio até as propriedades rurais. As distâncias médias percorridas por trecho (ida ou volta) para cada tipo 
Tabela 1 - Distância média percorrida por trecho para cada depósito de destino.

Table 1 - Average traveled distance for.

\begin{tabular}{ccc}
\hline Depósito & Veículo & Distância Média \\
\cline { 2 - 3 } & & $(\mathrm{Km})$ \\
\hline Acampamento & articulado & 102 \\
(AC) & $4 \times 2$ & 69 \\
& $6 \times 2$ & 80 \\
\hline Araguaia & $4 \times 2$ & 23 \\
(AR) & $6 \times 2$ & 30 \\
\hline Conceição da Barra & bitrem & 22 \\
(CB) & $4 \times 2$ & 40 \\
& $6 \times 2$ & 36 \\
\hline Fábrica & articulado & 41 \\
(FB) & $4 \times 2$ & 38 \\
& $6 \times 2$ & 53 \\
\hline Santa Teresa & $4 \times 2$ & 35 \\
(ST) & $6 \times 2$ & 25 \\
\hline
\end{tabular}

de ciclo operacional, ou seja, considerando as variáveis, tipo de veículo e depósito de destino, estão descritas na Tabela 1. A cadeia logística estudada não se estende ao transporte da madeira até a fábrica, pois essa atividade é de responsabilidade da empresa.

\subsection{Coleta de dados}

Os dados de tempo dos elementos do ciclo operacional, custos e movimentação de madeira no pátio foram coletados da Central de Logística da empresa e diretamente dos transportadores. Foram envolvidos na pesquisa o banco de dados da empresa e visita às áreas de estudo, para identificação e estruturação do carregamento de madeira no campo; levantamento da qualidade subjetiva das rodovias utilizadas; movimentação e descarregamento no pátio de estocagem; e controle dos tempos de execução das atividades do ciclo operacional do transporte da madeira de fomento.

\subsection{Qualidade subjetiva das rodovias}

Para classificação da qualidade da estrada florestal, é necessária a medição da geometria vertical (GV), que é a sinuosidade vertical da estrada medida em $\% / \mathrm{km}$; da geometria horizontal (GH), que é a sinuosidade horizontal da estrada expressa em graus $/ \mathrm{km}$; e do quociente de irregularidade (QI), que é a rugosidade do pavimento da estrada, expressa em mm/km. Para os índices de geometria foi utilizado um GPS (Global Position Satellite), que permite medir os ângulos das curvas horizontais, os aclives e os declives das estradas florestais. Enquanto

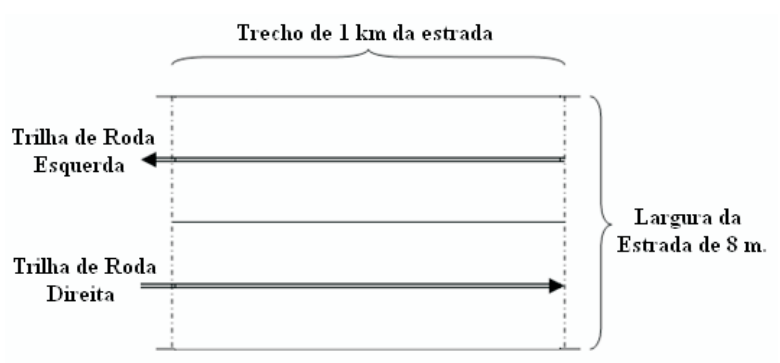

Figura 1- Esquema para medição do quociente de irregularidade das estradas florestais.

Figure 1 - Scheme for measurement of the forest road roughness index.

para mensurar o QI foram realizados o nivelamento e o contranivelamento, com o uso de um nível de precisão, a cada $20 \mathrm{~m} / \mathrm{km}$. Tendo como linha as trilhas de rodas direita e esquerda, que estão aproximadamente a $2 \mathrm{~m}$ de distância de cada margem, utilizou-se uma régua graduada para medir as irregularidades de acordo com o ponto de partida, como mostrado na Figura 1. Após o nivelamento, calculou-se o quociente de irregularidade utilizando o software VAROD. Dessa forma, os índices de geometria e quociente de irregularidade médio encontrados estão listados na Tabela 2 .

\subsection{Composições Veiculares de Carga (CVCs)}

O sistema de transporte rodoviário florestal utilizado era terceirizado, em sua grande maioria, mas também há transporte efetuado por veículos próprios dos produtores rurais fomentados, como mostrado na Tabela 3.

Entre as composições veiculares de carga utilizadas para esse transporte, são utilizados quatro tipos de veículos com carga líquida diferentes: a) veículo 4 x 2 , com capacidade de carga líquida de oito toneladas;

Tabela 2 - Índices médios de Geometria Vertical e Horizontal e Quociente de Irregularidade das estradas florestais utilizados para deslocamento da madeira até os referidos depósitos.

Table 2 - Average of the forest road Vertical and Horizontal Geometry Index and the Roughness Index used for the displacement of wood to the referred wood landing.

\begin{tabular}{|c|c|c|c|c|}
\hline Índices & Qualidade & GV & GH & QI \\
\hline Boa & & 15 & 120 & 90 \\
\hline Regular & & 28 & 240 & 150 \\
\hline Ruim & & 40 & 330 & 210 \\
\hline
\end{tabular}


Tabela 3-Distribuição e formas de transporte de madeira. Table 3 - Distribution and forms of wood hauling.

\begin{tabular}{llcccc}
\hline $\begin{array}{c}\text { Depósito } \\
\text { Transporte }(\%)\end{array}$ & AC & AR & CB & FB & ST \\
\hline Próprio & 31,2 & 31,2 & 0,0 & 14,8 & 10,0 \\
Terceirizado & 68,8 & 68,8 & 100,0 & 85,2 & 90,0 \\
\hline
\end{tabular}

b) veículo $6 \times 2$, com capacidade de carga líquida de 14 toneladas; c) veículo articulado, de cinco eixos e capacidade de carga líquida de 27 toneladas; e bitrem de sete eixos, com capacidade de carga líquida de 45 toneladas.

\subsection{Amostragem}

Para a coleta de dados dos ciclos operacionais de transporte rodoviário de madeira das áreas fomentadas, foi utilizado o processo de amostragem inteiramente aleatória. O número de ciclos operacionais amostrados no decorrer da coleta de dados foi estimado, utilizando-se a metodologia proposta por Barnes (1968).

$$
\text { em que: } \quad n \geq \frac{\mathrm{t}^{2}+\mathrm{CV}^{2}}{\mathrm{E}^{2}}
$$

n = número mínimo necessário de ciclos de transporte dos caminhões a serem amostrados;

$\mathrm{t}^{2}=$ valor de $\mathrm{t}$, para o nível de probabilidade desejado e $(\mathrm{n}-1)$ graus de liberdade;

$\mathrm{CV}=$ coeficiente de variação, em percentagem;e $\mathrm{E}=$ erro admissível, em percentagem.

O número de ciclos operacionais estimados foi de 27 e o número de cilcos operacionais amostrados, igual a 116 .

\subsection{Aplicação do modelo ADELCAPe dos programas JARPe ARP}

Utilizou-se o modelo ADELCAP para avaliação do desempenho logístico de cadeias produtivas, e os programas JARP e ARP como ferramentas de simulação interativa de redes, análise e avaliação da cadeia logística de transporte rodoviário de madeira de áreas de fomento da Aracruz Celulose com o uso de redes de Petri, para avaliar o seu desempenho e mensurar a melhoria do rendimento.

R. Árvore, Viçosa-MG, v.33, n.6, p.1159-1167, 2009
A ferramenta de modelagem utilizada neste modelo (Redes de Petri Temporais - RPT) serve para que sejam feitas análises sobre o sistema modelado baseadas nas propriedades das Redes de Petri, bem como simulações do sistema real. O modelo é, portanto: de simulação; dinâmico (já que os valores de saída dependem dos valores de entrada); determinístico (já que as variáveis de saída não são aleatórias); e contínuo (já que o tempo, principal variável de saída, é medido por números reais).

\subsubsection{Modelagem}

Modelo constitui representação simplificada de um sistema complexo para facilitar sua análise e seu projeto. Numa situação que envolve grande número de variáveis, tem seu comportamento influenciado por quantidade reduzida de variáveis principais. O relacionamento entre variáveis em um modelo pode ser representado na forma matemática de dois tipos:

a) Modelos de simulação que permitem a geração e análise de alternativas.

b) Modelos de otimização que não permitem a flexibilidade na escolha da alternativa, pois é estruturada para selecionar, segundo dado critério, a alternativa ótima.

A modelagem da cadeia produtiva do transporte rodoviário de madeira foi idealizada, de forma que a dinâmica da rede se torne análoga à de um processo qualquer de manufatura, e, para avaliar seu desempenho, foram utilizados os tempos dos elementos do ciclo operacional do transporte

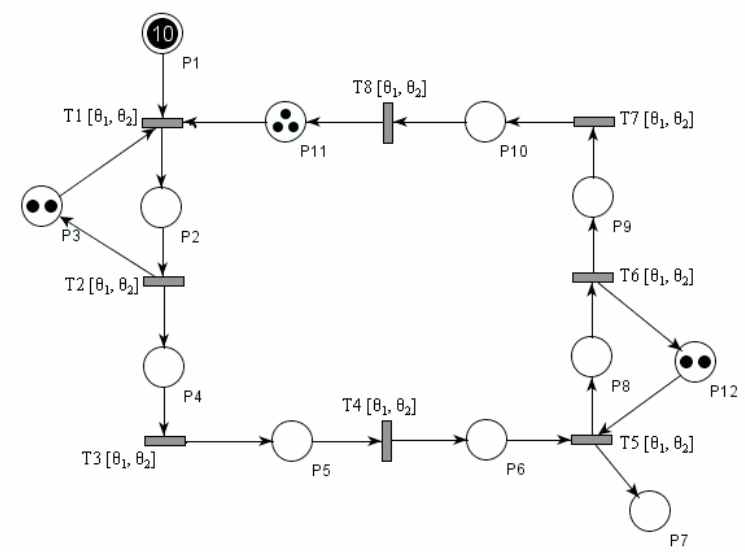

Figura 2 - Estrutura de Rede de Petri Temporal do transporte de madeira de áreas de fomento.

Figure 2 - Temporal Petri Network structure in a farm wood supply chain. 
Tabela 4 - Legenda da estrutura da Rede de Petri Temporal da cadeia logística do transporte rodoviário de madeira de áreas de fomento.

Table 4 - Legend of the Temporal Petri Network structure in a farm wood supply chain.

\begin{tabular}{l}
\hline LEGENDA \\
\hline Lugares: \\
P1 = Madeira cortada e processada, pronta para ser carregada \\
P2 = Veículo vazio sendo carregado \\
P3 = Carregador (manual e/ou mecanizado) livre para iniciar \\
carregamento \\
P4 = Veículo carregado pronto para iniciar deslocamento \\
P5 = Veículo carregado deslocando até o depósito \\
P6 = Veículo no depósito para iniciar descarregamento \\
P7 = Madeira descarregada no pátio do depósito \\
P8 = Veículo sendo descarregado no pátio do depósito \\
P9 = Veículo vazio pronto para retornar à propriedade rural \\
P10 = Veículo vazio deslocando até a propriedade rural \\
P11 = Veículo vazio na propriedade rural pronto para ser \\
carregado \\
P12 = Carregador mecanizado livre para iniciar descarregamento \\
Transições: \\
T1 = Início do carregamento \\
T2 = Carregamento do veículo \\
T3 = Início do deslocamento com o veículo carregado \\
T4 = Deslocamento com o veículo carregado \\
T5 = Início do descarregamento \\
T6 = Descarregamento do veículo \\
T7 = Início do deslocamento com o veículo descarregado \\
T8 = Deslocamento com o veículo descarregado
\end{tabular}

de madeira. O uso de Rede de Petri se adequa ao problema, permitindo análises de sensibilidade, verificação das propriedades do sistema e avaliação de seu desempenho com base no tempo do ciclo operacional do transporte rodoviário de madeira. Após essa etapa, procedeu-se à simulação computacional, utilizando a Rede de Petri como ferramenta de apoio à decisão, em nível operacional.

\subsubsection{Análise da estrutura e dos componentes da cadeia logística}

Foi realizado um diagnóstico da cadeia logística da empresa, com ênfase no seu desempenho, quanto aos tempos de ciclo operacional do transporte de madeira. O estágio atual (características, procedimentos e tecnologia empregados) de desenvolvimento da referida cadeia, bem como outros recursos de natureza logística, foi identificado e avaliado em relação ao desempenho em termos de tempo de ciclo. Dessa forma, estruturou-se graficamente a cadeia logística (Figura 2), segundo a legenda da Tabela 4.

\subsubsection{Elaboração dos cenários de aplicação do modelo}

Identificado o ciclo operacional da cadeia logística do transporte rodoviário de madeira, elaboraram-se os cenários, atuais e simulados, a serem investigados para cada depósito de acordo

Tabelas 5 - Cenários dos ciclos operacionais de transporte de madeira para cada depósito.

Table 5-Operational wood hauling cycles for each landing.

\begin{tabular}{|c|c|c|c|c|}
\hline \multicolumn{2}{|c|}{ Cenários } & \multirow[t]{2}{*}{ Veículo } & \multirow[t]{2}{*}{ Depósito } & \multirow{2}{*}{$\begin{array}{c}\text { Tipo de } \\
\text { Carregamento }\end{array}$} \\
\hline Atual & Simulado & & & \\
\hline A 1 & $\mathrm{H} 1$ & articulado & Acampamento & Manual \\
\hline A 2 & $\mathrm{H} 2$ & articulado & Acampamento & Mecanizado \\
\hline A 3 & H3 & $4 \times 2$ & Acampamento & Manual \\
\hline A4 & $\mathrm{H} 4$ & $4 \times 2$ & Acampamento & Mecanizado \\
\hline A5 & $\mathrm{H} 5$ & $6 \times 2$ & Acampamento & Manual \\
\hline A6 & H6 & $6 \times 2$ & Acampamento & Mecanizado \\
\hline A 7 & $\mathrm{H} 7$ & $4 \times 2$ & Araguaia & Manual \\
\hline A 8 & $\mathrm{H} 8$ & $6 \times 2$ & Araguaia & Manual \\
\hline A9 & H9 & bitrem & Conceição da Barra & Manual \\
\hline A 10 & $\mathrm{H} 10$ & bitrem & Conceição da Barra & Mecanizado \\
\hline A 11 & H11 & $4 \times 2$ & Conceição da Barra & Manual \\
\hline A 12 & H12 & $4 \times 2$ & Conceição da Barra & Mecanizado \\
\hline A 13 & H13 & $6 \times 2$ & Conceição da Barra & Manual \\
\hline A 14 & $\mathrm{H} 14$ & $6 \times 2$ & Conceição da Barra & Mecanizado \\
\hline A 15 & H15 & articulado & Fábrica & Manual \\
\hline A 16 & H16 & articulado & Fábrica & Mecanizado \\
\hline A 17 & H17 & $4 \times 2$ & Fábrica & Manual \\
\hline A 18 & H18 & $4 \times 2$ & Fábrica & Mecanizado \\
\hline A 19 & H19 & $6 \times 2$ & Fábrica & Manual \\
\hline A 20 & $\mathrm{H} 20$ & $6 \times 2$ & Fábrica & Mecanizado \\
\hline A 21 & $\mathrm{H} 21$ & $4 \times 2$ & Santa Teresa & Manual \\
\hline A 22 & $\mathrm{H} 22$ & $6 \times 2$ & Santa Teresa & Manual \\
\hline
\end{tabular}


com o tipo de veículo disponível, a forma de carregamento da madeira no veículo e a qualidade da estrada considerando as melhorias (Tabela 5).

De acordo com o Tabela 6, a velocidade e distância média percorrida em cada cenário atual foram calculadas e utilizadas como base comparativa para análise do desempenho logístico do transporte da madeira.

A análise do desempenho logístico do transporte levou em consideração a possibilidade de melhoria do tempo de deslocamento do veículo, seja ele carregado ou não. Os tempos mínimo e máximo obtidos nas coletas de dados do ciclo operacional atual para cada depósito, forma de carregamento da propriedade rural e tipo de veículo estão detalhados na Tabela 7 , assim como os tempos dos ciclos (minutos) a serem simulados, considerando-se a margem da velocidade média ( $\mathrm{km} /$ hora) melhorada de deslocamento.

\section{RESULTADOS E DISCUSSÃO}

\subsection{Diagnóstico do funcionamento atual da cadeia logística}

O cenário que representa a atual forma de operação do sistema modelado de transporte de madeira, da propriedade rural fomentada até o respectivo depósito

Tabela 6 - Qualidade da estrada e velocidade média dos veículos de transporte.

Table 6-Forest road quality and wood hauling vehicle average speed.

\begin{tabular}{|c|c|c|c|c|c|c|}
\hline \multirow[b]{2}{*}{ Depósito } & \multirow[b]{2}{*}{ Veículo } & \multicolumn{2}{|c|}{$\begin{array}{c}\text { Qualidade da estrada } \\
\text { florestal }\end{array}$} & \multicolumn{2}{|c|}{$\begin{array}{c}\text { Velocidade Média } \\
(\mathrm{km} / \mathrm{h})\end{array}$} & \multirow[t]{2}{*}{$\begin{array}{l}\text { Distância } \\
\text { Média }(\mathrm{km})\end{array}$} \\
\hline & & Atual & Melhorada & Atual & Melhorada & \\
\hline \multirow{3}{*}{$\mathrm{AC}$} & articulado & Regular & Boa & 49 & 72 & 102 \\
\hline & $4 \times 2$ & Ruim & Regular & 41 & 59 & 69 \\
\hline & $6 \times 2$ & Regular & Boa & 60 & 83 & 80 \\
\hline \multirow[t]{2}{*}{$\mathrm{AR}$} & $4 \times 2$ & Regular & Boa & 58 & 79 & 23 \\
\hline & $6 \times 2$ & Boa & Boa & 78 & 85 & 30 \\
\hline \multirow[t]{3}{*}{$\mathrm{CB}$} & bitrem & Regular & Boa & 41 & 64 & 22 \\
\hline & $4 \times 2$ & Ruim & Regular & 37 & 60 & 40 \\
\hline & $6 \times 2$ & Regular & Boa & 61 & 83 & 36 \\
\hline \multirow[t]{3}{*}{ FB } & articulado & Boa & Boa & 72 & 80 & 41 \\
\hline & $4 \times 2$ & Regular & Boa & 59 & 82 & 38 \\
\hline & $6 \times 2$ & Boa & Boa & 74 & 83 & 53 \\
\hline \multirow[t]{2}{*}{$\mathrm{ST}$} & $4 \times 2$ & Ruim & Regular & 40 & 56 & 35 \\
\hline & $6 \times 2$ & Regular & Boa & 63 & 84 & 25 \\
\hline
\end{tabular}

Tabela 7 - Tempos de cada atividade do ciclo operacional.

Table 7 - Activity of the operational cycle times.

\begin{tabular}{|c|c|c|c|c|c|c|c|c|c|}
\hline \multirow{2}{*}{$\begin{array}{c}\text { DEPÓSITO } \\
\text { Tempo }\end{array}$} & \multirow{2}{*}{$\begin{array}{l}\text { VEÍ́CULO } \\
\text { nutos) }\end{array}$} & \multicolumn{5}{|c|}{ ATUAL } & \multicolumn{3}{|c|}{ MELHORADO } \\
\hline & & $\begin{array}{l}\text { Carreg. } \\
\text { Manual }\end{array}$ & $\begin{array}{l}\text { Carreg. } \\
\text { Mecan. }\end{array}$ & $\begin{array}{l}\text { Viagem } \\
\text { Carreg. }\end{array}$ & Descar. & $\begin{array}{l}\text { Viagem } \\
\text { Descar. }\end{array}$ & $\begin{array}{l}\text { Viagem } \\
\text { Carreg. }\end{array}$ & $\begin{array}{l}\text { Viagem } \\
\text { Descar. }\end{array}$ & $\begin{array}{l}\text { Dist. } \\
\text { Média }\end{array}$ \\
\hline \multirow[t]{3}{*}{$\mathrm{AC}$} & articulado & $122-185$ & $53-60$ & $173-372$ & $48-61$ & $147-312$ & $70-173$ & $60-147$ & 102 \\
\hline & $4 \times 2$ & $60-73$ & $19-36$ & $101-230$ & $20-27$ & 86-195 & $25-101$ & $21-86$ & 69 \\
\hline & $6 \times 2$ & $91-123$ & $40-59$ & $101-229$ & $38-55$ & 86-195 & $36-101$ & $30-86$ & 80 \\
\hline \multirow[t]{2}{*}{ AR } & $4 \times 2$ & $62-73$ & $20-31$ & $29-72$ & $17-30$ & $24-60$ & $4-29$ & $3-24$ & 23 \\
\hline & $6 \times 2$ & $92-117$ & $44-64$ & $39-96$ & $41-60$ & $33-80$ & $4-39$ & $3-33$ & 30 \\
\hline \multirow[t]{3}{*}{$\mathrm{CB}$} & bitrem & $180-221$ & $73-89$ & $39-57$ & $67-84$ & $33-54$ & $26-39$ & $23-33$ & 22 \\
\hline & $4 \times 2$ & $59-71$ & $20-29$ & $71-87$ & $19-29$ & $60-72$ & $60-71$ & $45-60$ & 40 \\
\hline & $6 \times 2$ & $94-121$ & $44-63$ & $41-71$ & $39-59$ & $14-41$ & $35-60$ & $12-35$ & 36 \\
\hline \multirow[t]{3}{*}{ FB } & articulado & $119-176$ & $52-74$ & $54-157$ & $50-67$ & $27-54$ & $46-132$ & $23-46$ & 41 \\
\hline & $4 \times 2$ & $63-72$ & $22-33$ & $34-56$ & $19-31$ & $29-48$ & $28-53$ & $23-32$ & 38 \\
\hline & $6 \times 2$ & $91-120$ & $41-66$ & $55-144$ & $37-58$ & 46-120 & $21-55$ & $18-46$ & 53 \\
\hline \multirow[t]{2}{*}{ ST } & $6 \times 2$ & $60-75$ & $22-30$ & $47-84$ & $20-26$ & $40-47$ & $22-47$ & $19-40$ & 35 \\
\hline & $4 \times 2$ & $93-123$ & $39-61$ & $22-33$ & $35-53$ & $19-24$ & $18-22$ & $15-19$ & 25 \\
\hline
\end{tabular}

R. Árvore, Viçosa-MG, v.33, n.6, p.1159-1167, 2009 
de entrega de madeira, está representado por parte da Rede de Petri (Figura 2), através dos lugares $\left(\mathrm{P}_{\mathrm{x}}\right)$ e das transições $\left(\mathrm{T}_{\mathrm{x}}\right)$, e a partir desse cenário se processaram as simulações dos cenários hipotéticos.

\subsubsection{Simulação e resultados dos tempos dos ciclos operacionais por cenário}

Baseado na proposta de melhoria das estradas, de um nível de qualidade para outro (ruim - regular - boa), é certo que o tempo do ciclo operacional será reduzido, mas através das simulações é possível mensurar quanto afetará os resultados. Percebeu-se, nas regiões de topografia mais favoráveis, que a redução nos resultados são menores, já que os veículos conseguem desempenho melhor, mesmo quando as estradas não são de boa qualidade. Já nas regiões de relevo mais acidentado, onde os veículos já possuem maior dificuldade de deslocamento, os resultados simulados para deslocamento em estradas melhores foram mais satisfatórios (Tabela 8).

Nota-se nesse quadro que, através da melhoria das estradas, a redução do tempo do ciclo operacional para os cenários do depósito de Acampamento variou de 38,93\% a 53,22\%; de Araguaia, entre 30,36\% e 31,52\%; de Conceição da Barra, de 5,00\% a 14,36\%; da Fábrica, de $6,25 \%$ a $39,58 \%$; e de Santa Teresa, entre $5,97 \%$ a 22,61\% (Figura 3). As variações dos cenários referentes ao depósito de Acampamento apresentaram as maiores reduções, e isso se deveu ao fato de a região ser montanhosa e as estradas serem de baixa qualidade, comprometendo o desempenho dos veículos. Ao contrário,

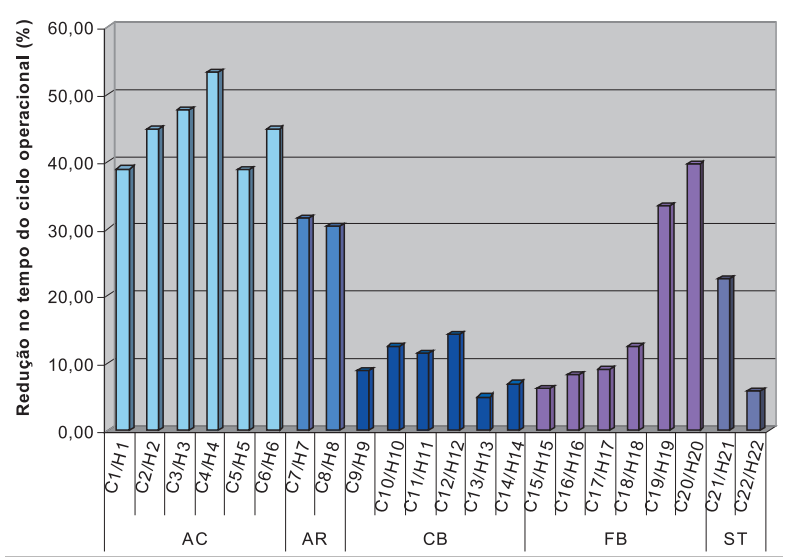

Figura 3-Impactos das reduções dos tempos dos ciclos operacionais.

Figure 3 - Reductions impacts of the operational cycles times.

Tabela 8-Média dos tempos dos ciclos operacionais e seus impactos.

Table 8 - Average of the operational cycle times and their impacts.

\begin{tabular}{|c|c|c|c|c|c|}
\hline$\overline{\text { Depósito }}$ & $\begin{array}{l}\text { Cenários } \\
\text { (Atuais) }\end{array}$ & $\begin{array}{l}\text { Tempo Médio } \\
\text { (minutos) }\end{array}$ & $\begin{array}{c}\text { Cenários } \\
\text { (Simulados) }\end{array}$ & $\begin{array}{l}\text { Tempo Médio. } \\
\text { (minutos) }\end{array}$ & $\begin{array}{c}\text { Variação Média } \\
\text { Atual/Simulado (\%) }\end{array}$ \\
\hline \multirow[t]{6}{*}{$\mathrm{AC}$} & A1 & 709 & $\mathrm{H} 1$ & 433 & $-38,93$ \\
\hline & A2 2 & 612 & $\mathrm{H} 2$ & 337 & $-44,93$ \\
\hline & A 3 & 396 & H3 & 207 & $-47,73$ \\
\hline & A4 & 357 & $\mathrm{H} 4$ & 167 & $-53,22$ \\
\hline & A5 & 459 & H5 & 281 & $-38,78$ \\
\hline & A6 & 402 & H6 & 222 & $-44,78$ \\
\hline \multirow[t]{2}{*}{$\mathrm{AR}$} & A7 & 184 & $\mathrm{H} 7$ & 126 & $-31,52$ \\
\hline & A 8 & 280 & $\mathrm{H} 8$ & 195 & $-30,36$ \\
\hline \multirow[t]{6}{*}{$\mathrm{CB}$} & A9 & 369 & H9 & 336 & $-8,94$ \\
\hline & A 10 & 248 & $\mathrm{H} 10$ & 217 & $-12,50$ \\
\hline & A 11 & 234 & H11 & 207 & $-11,54$ \\
\hline & A12 & 195 & $\mathrm{H} 12$ & 167 & $-14,36$ \\
\hline & A13 & 240 & H13 & 228 & $-5,00$ \\
\hline & A14 & 186 & H14 & 173 & $-6,99$ \\
\hline \multirow[t]{6}{*}{ FB } & A15 & 352 & H15 & 330 & $-6,25$ \\
\hline & A 16 & 267 & H16 & 245 & $-8,24$ \\
\hline & A 17 & 176 & H17 & 160 & $-9,09$ \\
\hline & A 18 & 136 & H18 & 119 & $-12,50$ \\
\hline & A19 & 335 & H19 & 223 & $-33,43$ \\
\hline & A 20 & 283 & $\mathrm{H} 20$ & 171 & $-39,58$ \\
\hline \multirow[t]{2}{*}{ ST } & A21 & 199 & $\mathrm{H} 21$ & 154 & $-22,61$ \\
\hline & A22 & 201 & $\mathrm{H} 22$ & 189 & $-5,97$ \\
\hline
\end{tabular}

R. Árvore, Viçosa-MG, v.33, n.6, p.1159-1167, 2009 
Tabela 9-Simulação de desempenho operacional do transporte de madeira $\left(\mathrm{t} \cdot \mathrm{km}^{-1} \cdot \mathrm{h}^{-1}\right)$.

Table 9 - Wood hauling operational performance simulation $\left(t . \mathrm{km}^{-1} \cdot \mathrm{h}^{-1}\right)$.

\begin{tabular}{|c|c|c|c|c|}
\hline Depósito & Cenários & Atual & Simulado & Veículo \\
\hline \multirow{6}{*}{ Acampamento } & 1 & 0,0224 & 0,0367 & articulado \\
\hline & 2 & 0,0259 & 0,0471 & articulado \\
\hline & 3 & 0,0176 & 0,0336 & $4 \times 2$ \\
\hline & 4 & 0,0195 & 0,0414 & $4 \times 2$ \\
\hline & 5 & 0,0229 & 0,0374 & $6 \times 2$ \\
\hline & 6 & 0,0261 & 0,0473 & $6 \times 2$ \\
\hline \multirow[t]{2}{*}{ Araguaia } & 7 & 0,1134 & 0,1656 & $4 \times 2$ \\
\hline & 8 & 0,1000 & 0,1436 & $6 \times 2$ \\
\hline \multirow[t]{6}{*}{ Conceição da Barra } & 9 & 0,3326 & 0,3653 & $\overline{\text { bitrem }}$ \\
\hline & 10 & 0,4949 & 0,5656 & bitrem \\
\hline & 11 & 0,0513 & 0,0580 & $4 \times 2$ \\
\hline & 12 & 0,0615 & 0,0719 & $4 \times 2$ \\
\hline & 13 & 0,0972 & 0,1023 & $6 \times 2$ \\
\hline & 14 & 0,1254 & 0,1349 & $6 \times 2$ \\
\hline \multirow[t]{6}{*}{ Fábrica } & 15 & 0,1123 & 0,1197 & $\overline{\text { articulado }}$ \\
\hline & 16 & 0,1480 & 0,1613 & articulado \\
\hline & 17 & 0,0718 & 0,0790 & $4 \times 2$ \\
\hline & 18 & 0,0930 & 0,1061 & $4 \times 2$ \\
\hline & 19 & 0,0473 & 0,0711 & $6 \times 2$ \\
\hline & 20 & 0,0560 & 0,0927 & $6 \times 2$ \\
\hline \multirow[t]{2}{*}{ Santa Teresa } & 21 & 0,0689 & 0,0891 & $4 \times 2$ \\
\hline & 22 & 0,1671 & 0,1778 & $6 \times 2$ \\
\hline
\end{tabular}

o transporte de madeira até o depósito de Conceição da Barra conta com a topografia favorável e distâncias menores a serem percorridas.

\subsubsection{Resultados do desempenho operacional por cenários}

Para avaliar o desempenho operacional do transporte de madeira em cada cenário, em função da carga transportada, distância percorrida e tempo de ciclo, foi calculado o desempenho em cada cenário simulado. Observou-se que o melhor desempenho do ciclo operacional está no cenário atual $\mathrm{C} 10$, consequentemente no simulado H10, devido ao fato de a distância média ser baixa $(22 \mathrm{~km}$ ) e de o relevo ser plano (planícies litorâneas), exigindo menos dos veículos e possibilitando o uso do bitrem, o que proporciona a melhor relação volume x distância x tempo (Figura 4 e Tabela 9).

Para o transporte de madeira de áreas de fomento florestal utilizando o veículo 4 × 2 e o 6 × 2, observouse que o fator de maior influência no desempenho foi a qualidade da estrada, visto que o transporte para o depósito de Araguaia, em região montanhosa, mas de qualidade regular, apresentou melhor desempenho que no depósito de Conceição da Barra, que é de região de planície, mas de qualidade ruim (Tabela 9).

R. Árvore, Viçosa-MG, v.33, n.6, p.1159-1167, 2009
No caso do veículo articulado, o relevo é o fator de maior influência. Para o transporte da madeira advinda das áreas de fomento que entregam no depósito de Acampamento, o desempenho do veículo pela relação $\mathrm{t} . \mathrm{km}^{-1} \cdot \mathrm{h}^{-1}$ foi de 0,0224 e 0,0259 , respectivamente, com carregamento manual e mecanizado, enquanto para o transporte até o depósito de Conceição da Barra, de relevo plano, apresentou desempenho de 0,1123 e $0,1480 \mathrm{t} \cdot \mathrm{km}^{-1} \cdot \mathrm{h}^{-1}$, respectivamente, com carregamento manual e mecanizado.

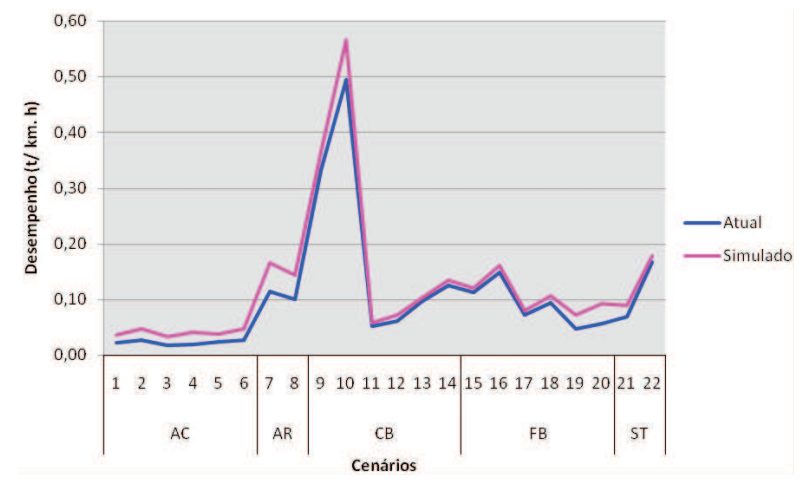

Figura 4 - Simulação do desempenho operacional do transporte de madeira nos diferentes cenários.

Figure 4-Operational performance simulation of the wood hauling for the differents sceneries. 


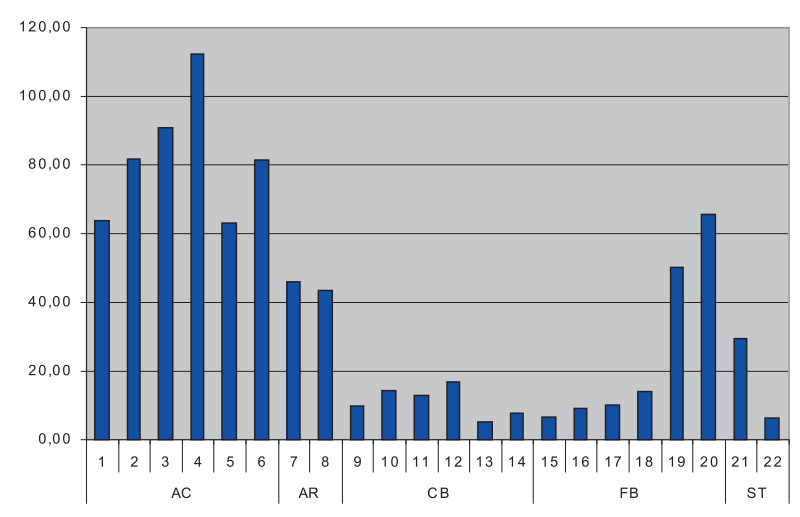

Figura 5 - Relação do desempenho operacional e melhoria nas estradas florestais.

Figure 5-Relation between the operational performance and forest road improvements.

Veículos com maior capacidade de carga líquida (articulado e o bitrem) aumentaram seu desempenho em função do relevo mais plano. Constatou-se que no transporte de madeira até o depósito de Conceição da Barra, com o uso de bitrem, mesmo em estradas de condição regular, apresentaram desempenho de 0,3326 $\mathrm{t} . \mathrm{km}^{-1} \cdot \mathrm{h}^{-1}$, quase três vezes maior que o desempenho do veículo articulado com topografia favorável e boa qualidade de estrada.

\subsubsection{Avaliação dos impactos em cada cenário}

O aumento do desempenho operacional do transporte de madeira está relacionado a topografia, escolha do tipo de veículo e qualidade das estradas. Na Figura 5, nota-se que em regiões montanhosas, como Acampamento e Araguaia, a melhoria das estradas geram maior impacto no desempenho dos veículos, pois os índices de qualidade são mais adversos e acarretam maior consumo de combustível, lubrificantes e pneus, entre outros, influenciando o aumento do custo do frete.

Nas regiões de planície como Fábrica e Conceição da Barra, apesar de as estradas terem qualidade um pouco melhor e a topografia não exigir tanto esforço mecânico do veículo, é possível realizar o ciclo operacional do transporte de madeira a uma velocidade maior, melhorando o desempenho dos veículos. Assim, percebeu-se que os melhores resultados de desempenho, com base na relação tonelada x quilômetro x tempo, seriam obtidos com os veículos de maior rendimento energético, ou seja, os articulados e os bitrens.

\section{CONCLUSÕES}

A Rede de Petri Temporal é uma ferramenta viável de modelagem, simulação, análise e avaliação de desempenho logístico do transporte rodoviário de madeira de áreas de fomento florestal.

Os principais fatores de influência no desempenho operacional do transporte de madeira das áreas de fomento são o relevo, a qualidade das estradas e o tipo de veículo.

Nas regiões montanhosas, a melhoria das estradas florestais tem maior influência para o aumento do desempenho dos veículos, em especial para os veículos de menor capacidade de carga útil.

Nas regiões de planícies, os veículos de maior capacidade de carga útil (articulado e bitrem) apresentaram melhor desempenho operacional, mostrando-se pouco sensíveis à qualidade das estradas florestais.

\section{AGRADECIMENTO}

Agradecemos ao CNPq, Fapemig e a Aracruz.

\section{REFERÊNCIAS}

BARNES, R. M. Motion and time study: design and measurement of work. 6.ed. New York: John Willey \& Sons, 1968. 799p.

CANTO, J. L. et al. Colheita e transporte florestal em propriedades rurais fomentadas no estado do Espírito Santo. Revista Árvore, v.30.n.6, p.989998, 2006. [online].

CHOPRA, S.; MEINDL, P., Gerenciamento da cadeia de suprimento. São Paulo: Pearson, 2004. 465p.

MACHADO, C. C. et al. Elementos básicos do transporte florestal rodoviário. Viçosa, MG: Universidade Federal de Viçosa, 2000. 167p.

MACHADO, R. R. et al. Avaliação do desempenho logístico do transporte rodoviário de madeira utilizando rede de petri em uma empresa florestal de Minas Gerais. Revista Árvore, v.30.n.6, p.9991008, 2006. [online].

VELLOSO, F. A. M.; LOPES, E. T.; ROLDI, L. M. "TRITREM" - Alternativa para o transporte de madeira. In: SIMPÓSIO BRASILEIRO SOBRE COLHEITAE TRANSPORTE FLORESTAL, 3., 1997, Vitória. Anais... Vitória: Sociedade de Investigações Florestais, 1997. p.157-175.

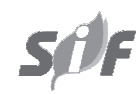

R. Árvore, Viçosa-MG, v.33, n.6, p.1159-1167, 2009 
